的投資方案的数学問題就是寻找所有的 $x_{i j}$, 使 $x_{i j}$ 滿足

$$
\frac{\sum_{i=1}^{m} x_{j}\left[\sum_{i=1}^{1} B_{i j}-\sum_{i=1}^{1} A_{i j}\right]}{\sum_{i=1}^{i} x_{j} A_{i}} \text { 为最大的条件。 }
$$

尽管在这里有大量的 $x_{i j}$, 但这个数学問题是可 以求得解答的。

上面說的这两个特殊的例于是很有用处的，因 为第一个特例是解决同类生产的規模間題，第二个 特例是解决近期效果与远期效果的梳一問題。

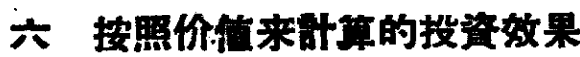

本文所討論的投资效果是从产品的使用价值来 考察的, 但是如果想研究从价值投入与生产的角度 来考察的投資效果，在方法上是完全相同的。只要 用投入的价值来代替投入的产品, 用生产出来的价 值来代替生产出来的产品, 用哹得价值来代替净产 品等等就可以了。而且在作这样的考察的还可以不 必附加捨缘了产品的特殊形态这个条件。但是如果 我們不只是从价值的投入与产出来考察投资效果， 而是把价格与价值可能背离的因素引入, 那么就不 能簢单地采取本文中所說的办法来謀求解决了。

\title{
环化聚合反应机构的研究
}

\section{張湍志 曹維孝馮新德}

自从 1953 年 Simpson ${ }^{[1]}$ 提出了具有两个非共䖝 双键的单体在聚合时可以发生环化現象以来, 环化 聚合的研究进展很快 ${ }^{[2-3]}$ 。1957 年 Butler[4] 等研究 二丙烯基第四胺盐的环化聚合后，提出了下远反应 机构的假定:

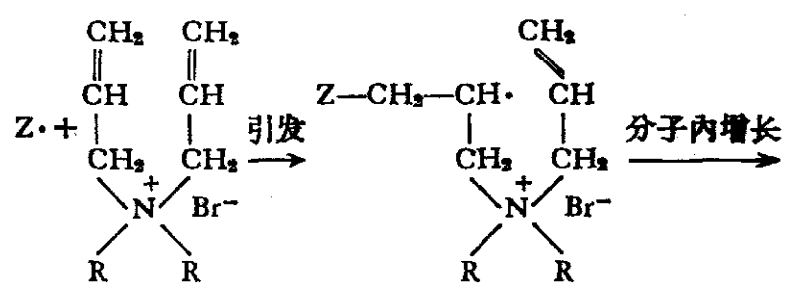

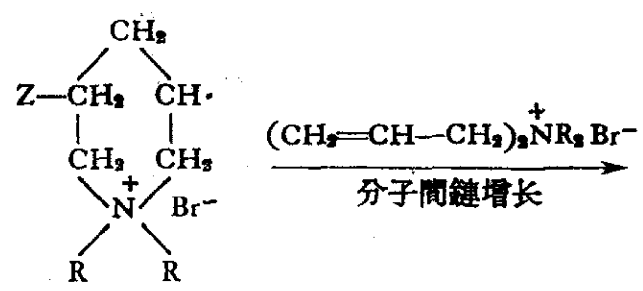

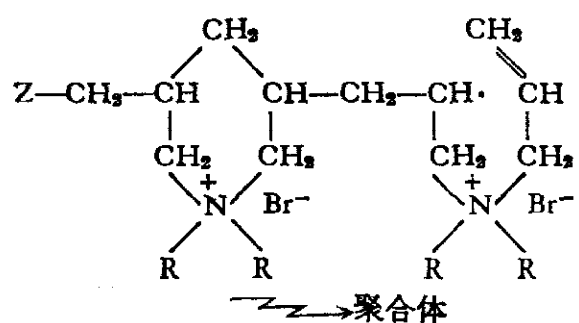

其中 $\mathrm{Z}$ 代表自由基， $\mathrm{R}$ 为脂肪或芳香烴基。

当自由基进攻单体时, 先使一个双踺打开, 再 經过分子內的环化，故分子的环化作用分先后两步 进行師所謂“分子內一一分子間”的反应机构（由于 这一反应机构是經过单分子的分子內环化一步, 因 此，我們簡称为“单分子环化机构”)。这一假定为 許多研究者所接受 ${ }^{[5-13]}$, 但都未能提供有力証据。

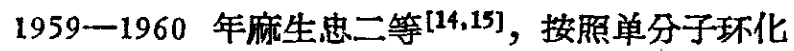
机构的假定, 应用一般共聚合的动力学理論, 推得 了环化聚合体的組分方程式:

$$
\frac{-d[\mathrm{~m}]}{d[\mathrm{M}]}=\frac{2 M_{0}}{2 M_{0}+r_{c}} \quad \text { (当 }[\mathrm{m}] \rightarrow 0 \text { 时) }
$$

其中 $[\mathrm{m}]$ 为聚合体中剩余双鍵浓度, 以[PDB]表示 （師“悬掛双鍵”，Pendent double bond），这是由于 
单体仅以一个双鍵帘加聚合 (非环化聚合) 的結果。 $[\mathrm{M}]$ 为单体浓度, $M_{0}$ 为单体起始浓度, $r_{c}$ 为反应 速率常数比。

应用这一方程式, 可以求得单体在不同起始浓 度时所得聚合体的环化百分率。但实驗結果表明， 随单体起始浓度变小，与理論值偏差加大。我們訩 为实驗結果与理論計算之間所以不符, 很可能是由 于麻生忠二等在理論推导时单一地采用单分子环化 机构所致。

1960 年 Butler ${ }^{[16]}$ 在討論为什么庚二烯 $-1,6$ 进 行聚合易于环化的問題时, 引用了 Mikulasova[12] 等的工作，扭䚯为由于庚二狶-1，6 分子內两个 双 键上电子相互作用而有成环趋势。我們訩为当具有 成环趋势的单体在受到自由基攻击时，也有可能分 子間鏈增长 (I) 与分子內鍕增长 (II)同步进行, 或者
叫做直接环化，成为双分子环化机构郎：
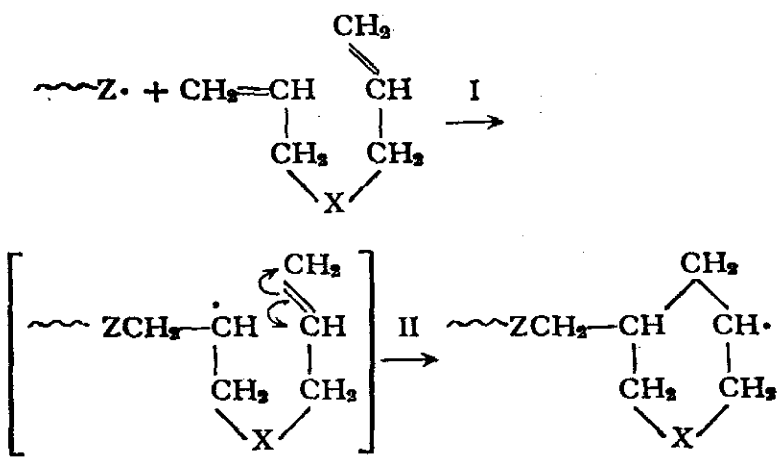

因此我們作了在环化聚合时除一般所知的单分子环 化机构外，上述的双分子环化机构、合扭考虑的 假 定。根据这一假定，我們进行了动力学推导如下。

对称的非共䡉双稀 $\left(\begin{array}{l}\mathrm{A} \\ \mathrm{A}\end{array}\right)$ 在鍕增长阶段的各种 可能反应:

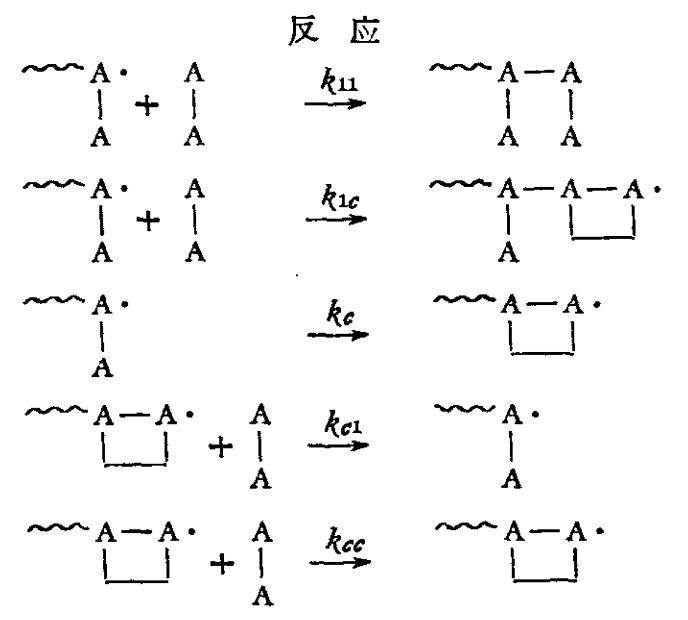

[m] (咱 [PDB]) 的增长速度:

$$
\begin{aligned}
\frac{d[\mathrm{~m}]}{d t}= & 2 k_{11}[\mathrm{M} \cdot][\mathrm{M}]+ \\
& +2 k_{c 1}[\mathrm{~m} \cdot][\mathrm{M}]-k_{c}[\mathrm{M} \cdot]
\end{aligned}
$$

$[\mathrm{M}]$ 的消失速度:

$$
\begin{aligned}
\frac{-d[\mathrm{M}]}{d t} & =2 k_{11}[\mathrm{M} \cdot][\mathrm{M}]+2 k_{1 c}[\mathrm{M} \cdot][\mathrm{M}]+ \\
& +2 k_{c 1}[\mathrm{~m} \cdot][\mathrm{M}]+2 k_{c c}[\mathrm{~m} \cdot][\mathrm{M}]
\end{aligned}
$$

在稳态时得

$$
\frac{-d[\mathrm{M}]}{d[\mathrm{~m}]}=A+\frac{B}{[\mathrm{M}]}
$$

其中

$$
A=1+\frac{k_{1 c}\left(k_{c 1}+k_{c c}\right)}{k_{c 1}\left(k_{11}+k_{1 c}\right)}
$$

聚合体結构自由基变化 反应速度

有 PDB 生成 $\quad \mathrm{M} \cdot \longrightarrow \mathrm{M} \cdot 2 k_{11}[\mathrm{M} \cdot][\mathrm{M}]$

难分子环化
PDB 生成

单分于环化， 无 PDB 生成

$\mathrm{M} \cdot \longrightarrow \mathrm{m} \cdot \quad k_{c}[\mathrm{M} \cdot]$

$\mathrm{m} \cdot \longrightarrow \mathrm{M} \cdot \quad 2 k_{c 1}[\mathrm{~m} \cdot][\mathrm{M}]$

双分子环化 无 PDB 生成

$\mathrm{m} \cdot \longrightarrow \mathrm{m} \cdot \quad 2 k_{c e}[\mathrm{~m} \cdot][\mathrm{M}]$
（9）式在低轉化率时， $\frac{-d[\mathrm{M}]}{d[\mathrm{~m}]}=\frac{\text { 单体进入聚合体分子总数 }}{\text { 聚合体中 PDB 分子总数 }}=$ $=\frac{1}{\text { 聚合体中 PDB 分子分数 }}$

聚合体中 PDB 分子分数是可以測定的, 故 $\frac{-d[\mathrm{M}]}{d[\mathrm{~m}]}$ 值可以由实騟測得, 作 $\frac{-d[\mathrm{M}]}{d[\mathrm{~m}]}$ 对 $\frac{1}{[\mathrm{M}]}$ 图应得一直

䋐，其截距为 $A$, 斜率为 $B$ 。 年外，由(3)、(4)、(6)式， 


$$
\begin{aligned}
& \text { 双分子环化反应速度 } \\
& \text { 单分子环化反应速度 } \\
& =\frac{2 k_{c c}[\mathrm{~m} \cdot][\mathrm{M}]+2 k_{1 c}[\mathrm{M} \cdot][\mathrm{M}]}{k_{c}[\mathrm{M} \cdot]}
\end{aligned}
$$

在稳态时:

$$
\begin{aligned}
& \left.\frac{\text { 双分子环化反应速度 }}{\text { 简分称难环化反应速度 }}\right)= \\
& =\frac{k_{c c}}{k_{c 1}}+2 \frac{k_{s c}}{k_{c 1}} \frac{k_{1 c}}{k_{c}}[\mathrm{M}]+2 \frac{k_{1 c}}{k_{c}}[\mathrm{M}]
\end{aligned}
$$

其中 $k_{c c}, k_{c 1}, k_{1 c}$ 都是正数，故

$$
\text { 䛨 }>2 \frac{k_{1 c}}{k_{c}}[\mathrm{M}] \quad\left(=\frac{A-1}{B}[\mathrm{M}]\right)
$$

所以，由实驗求得 $A, B$ 值后，可以定性 地看出在 这个环化聚合中双分子及单分子环化机构例个較为 重要。

由(9)式也可以看出:

（i）若只考虑单分子环化机构, 則

$$
k_{1 c}=0, \quad k_{c c}=0
$$

(9)式化为

$$
\frac{-d[\mathrm{M}]}{d[\mathrm{~m}]}=1+\frac{k_{n}}{2 k_{11}} \cdot \frac{1}{[\mathrm{M}]}
$$

这也就是麻生忠二所得的公式 $[$ 見(1)式 $]$,作一 $\frac{d[\mathrm{M}]}{d[\mathrm{~m}]}$ 对 $\frac{1}{[\mathrm{M}]}$ 图, 所得直紷的截距永远通过“ 0,1 ”点。

（ii）若只考虑双分子环化机构, 則

$$
\begin{aligned}
& k_{c}=0, \quad(9) \text { 式化为 } \\
& \frac{-d[\mathrm{M}]}{d[\mathrm{~m}]}=A=\text { 常数 }
\end{aligned}
$$

即 $\frac{-d[\mathrm{M}]}{d[\mathrm{~m}]}$ 与单体起始浓度无关, 或若以 $\frac{-d[\mathrm{M}]}{d[\mathrm{~m}]}$ 对 $\frac{1}{[\mathrm{M}]}$ 作图所得直馢其斜率 $(B)$ 为零。

我們做了二丙烯基丙二酸二乙酯 (DDM) ${ }^{[19]}$ 及 邻苯二酸二丙烯酯 (DAP) 的环化聚合 (图 1,2)。

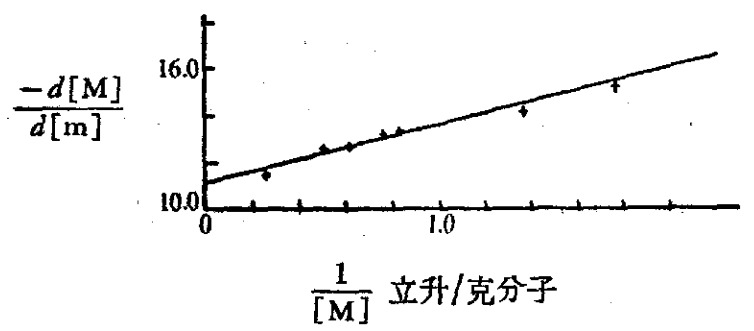

图 $1 \mathrm{DDM}$ 的环化聚合 $\frac{-d[\mathrm{M}]}{d[\mathrm{~m}]} \sim \frac{1}{[\mathrm{M}]}$

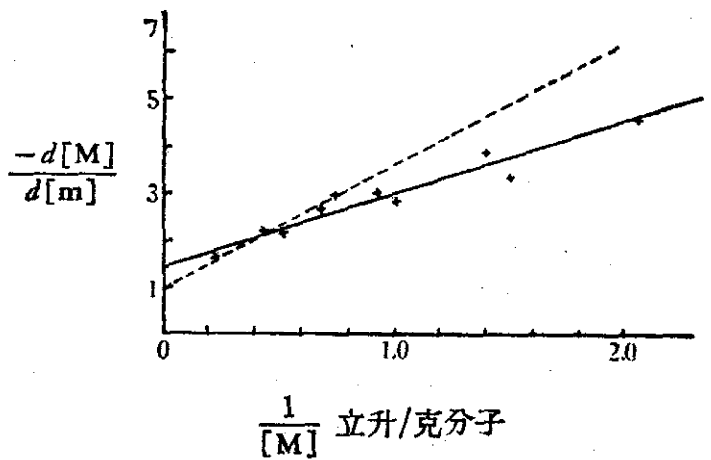

图 2 DAP 的环化聚合 $\frac{-d[\mathrm{M}]}{d[\mathrm{~m}]} \sim \frac{1}{[\mathrm{M}]}$

另外，我們对麻生忠二[15] 的二甲基丙堵酸二縮乙二 醇酯 (DDMA) 环化聚合的实驗結果也进行了处理, 得图 3。

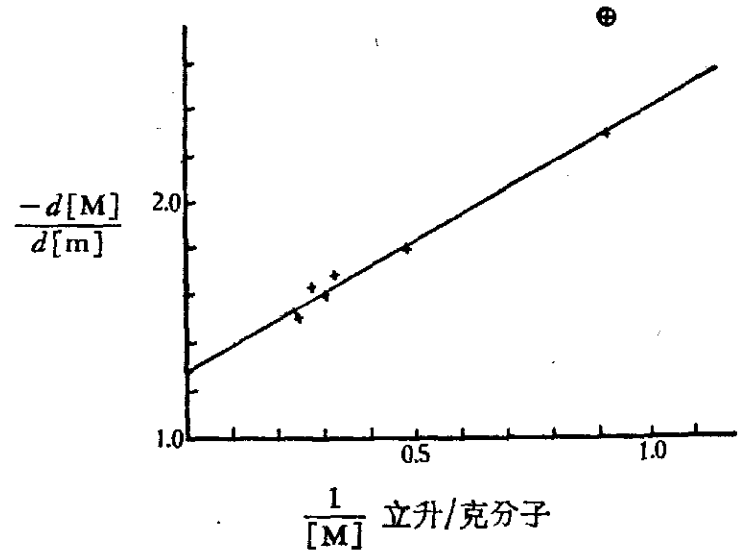

图 $3 \mathrm{DDMA}$ 的环化聚合 $\frac{-d[\mathrm{M}]}{d[\mathrm{~m}]} \sim \frac{1}{[\mathrm{M}]}$

图中 $\oplus$ 为由筑生忠二的 $\frac{-d[\mathrm{M}]}{d[\mathrm{~m}]}=\frac{2 M_{0}}{2 M_{0}+r_{c}}$

公式, 当 $r_{c}=4.0$ 部算面得。

在三种情况下，实驗数据基本上符合 (9) 式的 直綫关系 (图 1,2，3)。从截距来看, 三种情况都 不等于 “ 1 ”，特別对 DDM，其截距为 11.1 (图 1) 与 “ 1 ” 相差蔍远。說明只考虑单分子环化机构所得 的 (13)式是与实驗結果不符合的。我們将 DAP 的 实驗数据按 (13)式作图, 即使直綫通过 “ 0,1 ” 点, 在最好的情况下得图 2 的虚䋐, 在浓度較大时晌能 与实驗結果符合，浓度愈小，偏差愈大（这与麻生 忠二 ${ }^{[14 ， 15]}$ 对 DDMA 石二甲基两烯酸乙二醇酯研究 时出現偏差情况相似)。換言之，若将实驗値代入 (13)式則結果 $\frac{k_{c}}{k_{11}}$ 为变数, 亦師麻生忠二的 (1)式 
中的 $r_{c}$ 为变数。若按 $(9)$ 式, 則一直到 $[M]=0.478$ 克分子/立升时，侣能符合䋨性关系。对DDM, 則根 本无法使直馢通过 $(0,1)$ 点 (图 1)。在 DDMA 的 情况 (图 3)，若按麻生忠二的意見，在 (1) 式中以 $r_{c}=4.0$ 計算, 則当 $\frac{1}{[\mathrm{M}]}$ 为 0.91 时, $\frac{-d[\mathrm{M}]}{d[\mathrm{~m}]}$ 为 2.82 , 实驗値是 2.32 , 偏差較大(如图 3 所示)。因 此我們䚯为上拈三种情况都說明了只考虑单分子环 化机构是不全面的。另外，三条直綫的斜率都不是 零(在 $\mathrm{DDM}$ 为 $2.48, \mathrm{DAP}$ 为 $1.45, \mathrm{DDMA}$ 为 1.17 )。 这說明也不能只考虑双分子环化机构。把单分子 与奴分子机构結合起来考虑, 則結果与实驗基本相 符。

我們进一步利用 (12)不等式定性地了解在两种 环化机构中哪种更重要些。所得結果列为表 1 。在 DDM 本体聚合时，环化机构按双 分子比按 单分子 至少快 16.3 倍; 在 DAP 及 DDMA 情况下，至少双 分子反应机构不比单分子反应机构来得次要，所以 双分子机构在三种情况下都是不容忽視的。

表 1 按两种环化机构反应速度比

\begin{tabular}{l|c|c|c}
\hline 单 体 & A & B & 器 (本体聚合) \\
\hline DDM & 11.1 & 2.48 & $>16.3$ \\
DAP & 1.42 & 1.45 & $>1.27$ \\
DDMA & 1.27 & 1.17 & $>0.97$ \\
\hline
\end{tabular}

非共䡉双烯的这种能够按双分子机构进行环化 聚合的現象，引起了我們对非共䡉双烯在单体状态 时两个双鍵上的电子云相互作用 ${ }^{[16]}$ 的重視。我們 对 DDM 及一丙烯基丙二酸二乙酯(DMM)照摄了紅 外吸收光譜。这两种单体应在碳一碳双鍵特征吸收 䋅的波数上表現不同。实驗結果: 用三种不同 度的 DDM 四氧化碳溶液測得的波数, 严格重复在

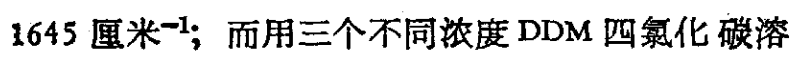
液測得結果則严格重复在 1647 厘米 ${ }^{-1}$ 。二者吸收 䟖波数有偏离, 但不大, 只有 2 厘米 ${ }^{-1}$ 。实驗仪器
可能产生的最大誤差为 \pm 1 厘米 ${ }^{-1}$ 。从其波数严格 可重复性来看, 这 2 重米 ${ }^{-1}$ 的偏离虽小，但不是可 以用实驗誤差去解释的。Marvel 等 ${ }^{[18]}$ 在庚二烯-1,6 的数据中，其碳一碳双鍵特征吸收峯在 1647 厘米 ${ }^{-1}$ 处; 而在聚庚二烯 $-1,6$ (有 PDB) 中則为 1650 厘 米 $^{-1}$ 。显然, 其单体分子內成环趋势与聚合体时有 很大不同, 在紅外吸收光諎上也显示了微小的偏离 (3 厘米 $\left.{ }^{-1}\right)$ 。这和我們在 DDM 及 DMM 的紅外光 譜上所覌察到的現像相似 ${ }^{[19]}$ 。这种微小的偏 或 都就是由单体状态时双鍵电子云相互作用而有成环 趋势引起，但倘需作更进一步的研究。

[1] W. Simpson, J. Polymer Sci., 10, 489, 1953.

[2] 䂇德暗, 化学通报, 3 期 1 頁, 1962 年。

[3] Г. С. Колесников, С. Л. Давыдова, Успехп Химид, 29, 1474, 1960.

[4] G. B. Butler, R. ]. Angelo, J.A.C.S., 79, 3128, 1957.

[5] C. S. Marvel, R. G. Vest, ibid., 79, 5771, 1957.

[6] 大岩正芳、尾形弥生, 日本化学杂志, 39, 475, 1957。

[7] С. Г. Мацоян, М. Г. Аветян, М. Г. Восканян, Высоком. Соед., 3, 562, 1961.

[8] 三宅泰治, 工业化学杂志 (日本), 64, 359, 1961。

[9] H. Rigsdorf, C. G. Overberg, Makromolek, Chem., 44-46, 423, 1961.

[10] J. F. Jones, J. Polymer Sci., 33, 15, 1958.

[11] K. D. Belin, G. B. Butler, J.A.C.S., 82, 2712 , 1960.

[12] D. Mikulasova, A. Hrivik, Chem, Zuesti., 11, 641, 1957.

[13] W. E. Gibls, et al., J. Polymer Sci., 54, No. 159, S1, 1961.

[14] Chuji Aso, ibid., 39, 475, 1959.

[15] 麻生忠二、貞方一夫, 工业化学杂志 (日本), 63, 188,1960 .

[16] G. B. Butler, J. Polymer Sci., 48, 279, 1960.

[17] M. D. Barnett, A. Crawshow, G. B. Butler, J.A.C.S., 81, 5946, 1959.

[18] C. S. Marvel, J. K. Stille, J.A.C.S., 80, 1742, 1958.

[19] 馮新德、张潟志、曹維孝、沈 鹏、习复、暲䥀 芝、紀雪桥，科学通报，第 5 期，53 頁，1963。 